\title{
CUANTIFICACIÓN DE RESIDUOS SÓLIDOS URBANOS GENERADOS EN LA CABECERA MUNICIPAL DE BERRIOZÁBAL, CHIAPAS, MÉXICO
}

\author{
Juan Antonio ARAIZA AGUILAR ${ }^{1 *}$, Juan Carlos CHÁVEZ MORENO² y \\ José Ariosto MORENO PÉREZ ${ }^{2}$
}

${ }^{1}$ Instituto de Geografía, Universidad Nacional Autónoma de México. Circuito exterior, Ciudad Universitaria, Delegación Coyoacán, Ciudad de México, México, C. P. 04510

${ }^{2}$ Escuela de Ingeniería Ambiental, Universidad de Ciencias y Artes de Chiapas. Libramiento Norte-Poniente. Colonia Lajas Maciel, Tuxtla Gutiérrez, Chiapas, México, C. P. 29000

*Autor para correspondencia: araiza0010@hotmail.com

(Recibido agosto 2016; aceptado abril 2017)

Palabras clave: caracterización, generación, muestreo, subproductos

\section{RESUMEN}

Los estudios de generación y cuantificación de subproductos de los residuos sólidos urbanos, proporcionan herramientas base para la toma de decisiones en los municipios y en las localidades de México. Este trabajo fue realizado en la cabecera municipal de Berriozábal, Chiapas. Como resultados se determinó que la generación per cápita de residuos sólidos urbanos es de $0.619 \mathrm{~kg} / \mathrm{hab} / \mathrm{d}$, la fracción doméstica equivale a $0.456 \mathrm{~kg} / \mathrm{hab} / \mathrm{d}$ y la no doméstica a $0.160 \mathrm{~kg} / \mathrm{hab} / \mathrm{d}$. Respecto a la composición, se encontró que la fracción orgánica sigue manteniendo porcentajes altos $(54.88 \%)$, seguido de otros subproductos como plásticos $(11.70 \%)$, además de papel y cartón $(6.87 \%)$. Poco más de $78 \%$ podría ser considerado como susceptible de recuperación, por lo que podrían ser aprovechados de alguna manera para no enviarlos directamente a disposición final y con ello alargar la vida útil de los sitios de depósito de residuos.

Key words: characterization, generation, sampling, subproducts

\begin{abstract}
The studies of generation and characterization of solid wastes provide basic tools for decision-making in municipalities and towns of Mexico. This study carried was out in the municipal seat of Berriozabal, Chiapas. It was determined that the per capita generation of municipal solid waste is $0.619 \mathrm{~kg} / \mathrm{hab} / \mathrm{d}$, the per capita generation for domestic sources is $0.456 \mathrm{~kg} / \mathrm{hab} / \mathrm{d}$, and $0.160 \mathrm{~kg} / \mathrm{hab} / \mathrm{d}$ for no domestic sources. Regarding the composition, the organic fraction continues to maintain high percentages $(54.88 \%)$, followed by other products such as plastics $(11.70 \%)$, plus paper and cardboard $(6.87 \%)$. Over $78 \%$ of solid waste generated could be used in some way, so it would not be send directly to final disposal, thus extending the lifetime of the waste disposal sites.
\end{abstract}




\section{INTRODUCCIÓN}

Los residuos sólidos urbanos (RSU) son definidos en el marco de la legislación mexicana como:

"Los generados en las casas habitación, que resultan de la eliminación de los materiales que utilizan en sus actividades domésticas, de los productos que se consumen y de sus envases, embalajes o empaques; así también son los que provienen de cualquier otra actividad dentro de establecimientos o en la vía pública que genere residuos con características domiciliarias y los resultantes de la limpieza de las vías y lugares públicos" (DOF 2014).

La generación de RSU está ligada a las actividades antrópicas que el ser humano desarrolla diariamente y es maximizada por diversos factores como el crecimiento de la población, los cambios en los hábitos de consumo, la migración o las nuevas costumbres. Estos factores ocasionan que el manejo de residuos represente un gran desafío y una problemática actual para la sociedad (Buenrostro y Bocco 2003, GTZ 2003, Ojeda y Beraud 2003).

Un método que permite estimar la cantidad de residuos que producen las localidades urbanas y rurales, así como cuantificar el equipamiento necesario (camiones recolectores y contenedores) y diseñar las obras de ingeniería (rellenos sanitarios y plantas de separación), son los llamados estudios de generación y cuantificación de subproductos de los RSU (Bernache et al. 2001, Al-Jarallah y Aleisa 2014).

En el contexto nacional este tipo de estudios son ampliamente utilizados, aunque no frecuentemente divulgados. Se destacan los realizados por la $\mathrm{Se}-$ cretaría de Desarrollo Social entre 1980 y 1990 en diversas zonas del país, con cifras compiladas en los documentos del INE (1997) de INE-SEMARNAT (1999/2006). Además de otros trabajos de investigación como los realizados por Fierro et al. (2010), Aguilar et al. (2010) y Castillo y De Medina (2014), tanto en centros comerciales, pequeñas localidades urbanas y en los propios sitios de depósito de RSU respectivamente.

En el estado de Chiapas por otro lado, se han publicado algunos trabajos como los de Esquinca et al. (1996), Villalobos (1996), Esquinca et al. (1997), Escamirosa et al. (2001) Alvarado et al. (2009) y Araiza et al. (2015), quienes realizaron estudios de generación o diagnósticos de manejo de RSU en diversos municipios (cabeceras municipales usualmente) como Tuxtla Gutiérrez, Las Margaritas, Huixtla, Mapastepec, Arriaga, Tonalá, entre otros. Aunque existe todavía un vacío de información, dado los 122 municipios que conforman la entidad chiapaneca.
Una parte significativa de los trabajos publicados en el estado, fueron generados por la Secretaría de Ecología, Recursos Naturales y Pesca (SERNYP), hoy Secretaría de Medio Ambiente e Historia Natural (SEMAHN), quienes han dado las bases para la aparición de este tipo de trabajos. Otra porción de trabajos no publicados en forma de artículos, pertenecen a tesis de obtención de grado, como los de Carrera (2012), Cabrera (2013) y Reyes (2016), aunque generalmente son elaborados en localidades pequeñas de menos de 2500 hab, estimando únicamente la generación de residuos domésticos.

En este sentido, el presente trabajo muestra los resultados de un estudio de generación y cuantificación de subproductos de los RSU, elaborado en la cabecera municipal de Berriozábal, Chiapas. Lo anterior con la intención de que la información presentada pueda ser empleada por los tomadores de decisiones dentro de los municipios. Adicionalmente, esta información podrá servir a los docentes e investigadores de instituciones educativas en sus actividades académicas, ya sea para su análisis, enseñanza e inclusive, para mejorar o implementar futuros programas sobre la recolección y el tratamiento de RSU en el municipio.

\section{MATERIALES Y MÉTODOS}

\section{Descripción del área de estudio}

El municipio de Berriozábal, Chiapas, México, se ubica en la región socioeconómica denominada metropolitana, en las coordenadas geográficas $16^{\circ} 48^{\prime} \mathrm{N}$ y $93^{\circ} 17^{\prime} \mathrm{W}$ (Fig. 1). Es el segundo municipio más grande de la región con una extensión territorial de $353.23 \mathrm{~km}^{2}$. Su población en 2010 fue de $43179 \mathrm{hab}$ (52675 hab, cifra estimada para 2016 por los autores de este trabajo), distribuidas en 306 localidades, donde sólo la Cabecera Municipal y la localidad de Ciudad Maya son de tipo urbanas, pues habitan en ellas $65.1 \%$ de la población total (SHCP 2011).

Como en muchas de las ciudades del país, en los últimos años la cabecera municipal de Berriozábal ha experimentado un crecimiento en su población de manera notoria, que se refleja en la edificación de nuevos conjuntos habitacionales. Lo que ha repercutido en la generación de RSU, ya que además de haber aumentado el número de colonias, también se han ampliado el número de establecimientos comerciales y de servicios, específicamente en la zona centro de la cabecera o primer cuadro de la misma. 

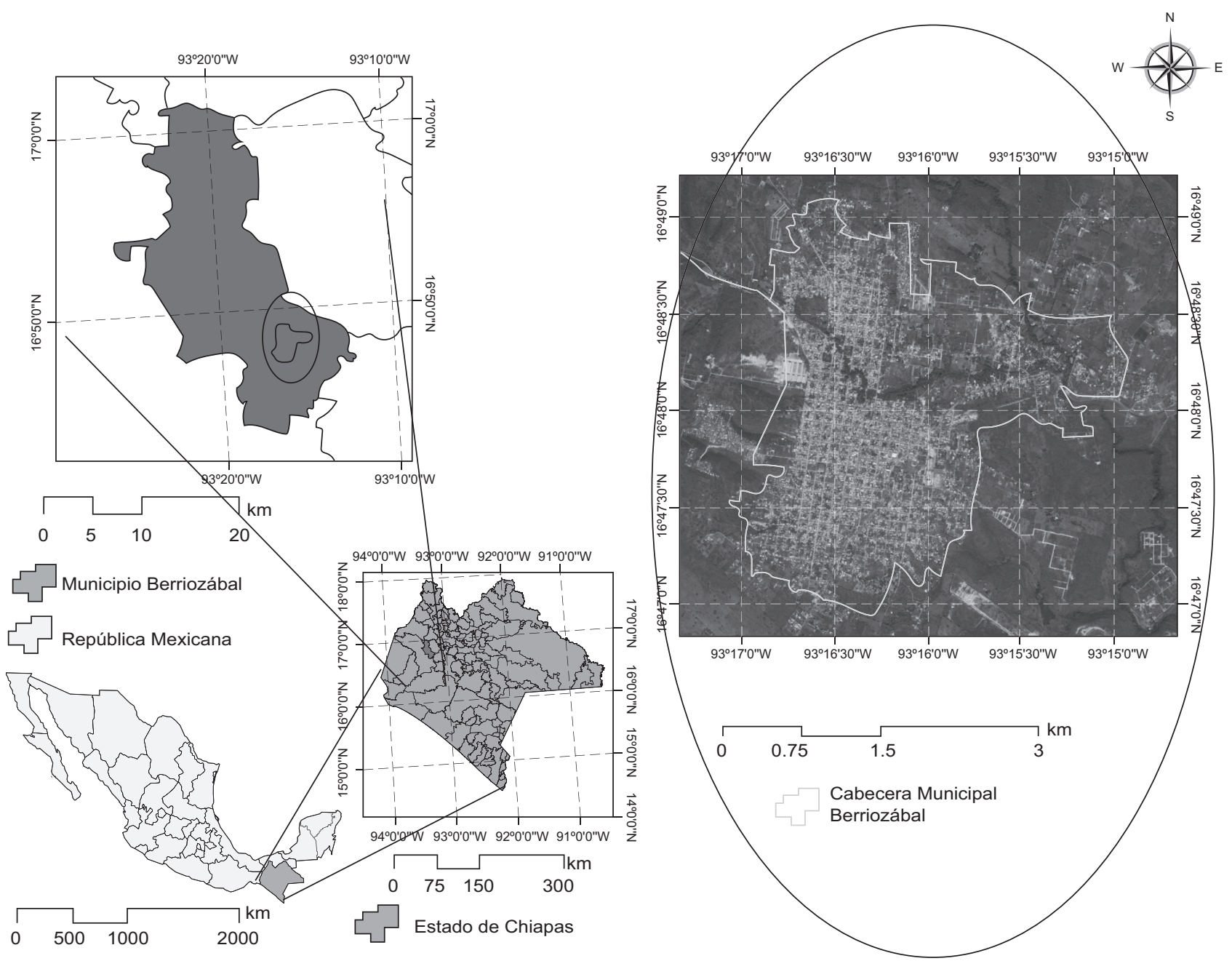

Fig. 1. Área de estudio

\section{Recopilación de información previa}

En esta fase se adquirió información de tipo cartográfico (planos de catastro y manzanas) y documental (anuario estadístico), tomados de las bases de datos del Instituto Nacional de Estadística y Geografía (INEGI) y del H. Ayuntamiento municipal. Con dicha información, se estableció un único estrato socioeconómico dentro de la cabecera municipal.

Respecto a la generación no doméstica de residuos (comercial y de servicios), se emplearon bases de datos o cuadros de generación de residuos por tipo de establecimiento, específicamente las publicadas en Heredia et al. (2007) y Alvarado et al. (2009). Asimismo, se utilizó la información del Directorio Estadístico Nacional de Unidades Económicas (DENUE) del INEGI (2016) para identificar el número de establecimientos dentro de la cabecera municipal de Berriozábal.

\section{Selección de muestras}

Con el apoyo del plano de catastro proporcionado por el $\mathrm{H}$. ayuntamiento, se numeraron las viviendas y con los números aleatorios arrojados por un programa estadístico, se seleccionaron 115 casas como premuestras, de acuerdo con lo que indica la norma NMX-AA-061-1985 (SECOFI 1985d) para trabajar con un nivel de confianza de $95 \%$.

La identificación, explicación y entrevistas a los jefes de familia de las viviendas participantes comenzó el 12 de julio de 2016. Posterior a la entrevista y aceptación, se les hizo entrega de una bolsa de polietileno, la cual era llenada con los residuos generados en la casa habitación y recolectada al día siguiente para su posterior análisis. Esta operación se repitió hasta el 19 de julio de 2016 en que finalizó el estudio.

Es importante mencionar que el primer día de colecta de las bolsas, éstas no fueron pesadas y 
analizadas, sino que únicamente se recolectaron con la finalidad de evitar sesgos en el muestreo.

\section{Procedimiento de recolección y análisis de los residuos sólidos domésticos}

Una vez recolectadas las bolsas en las viviendas seleccionadas, éstas fueron llevadas al espacio de trabajo designado por el $\mathrm{H}$. Ayuntamiento, en donde se registró el peso de cada bolsa en una base de datos.

Posterior al pesaje, los residuos en cada bolsa se vaciaron y homogeneizaron de acuerdo con lo indicado en la norma NMX-AA-015-1985 (SECOFI 1985a). De acuerdo con la norma NMX-AA-019-1985 (SECOFI 1985b) se determinó el peso volumétrico de los residuos a través de un recipiente de $200 \mathrm{~L}$.

Para realizar la caracterización o cuantificación de subproductos, una porción de aproximadamente 50 $\mathrm{kg}$ obtenida por el método de cuarteo fue empleada. Los residuos eran colocados sobre una mesa, mismos que se caracterizaron con base en las fracciones y componentes enlistadas en el cuadro I, además de lo indicado por la norma NMX-AA-022-1985 (SECOFI 1985c).

\section{RESULTADOS Y DISCUSIÓN}

\section{Análisis estadístico en fuentes domésticas}

De las 115 viviendas pre-seleccionadas, únicamente se logró trabajar con 91, mismas que participaron en la entrega de muestras con residuos durante todo el periodo de estudio ( 7 días de recolección regular y 1 día de operación limpieza). Posterior a los pesajes, obtención de pesos volumétricos y caracterización de residuos de los siete días de trabajo, se examinó la base de datos con un análisis de exclusión de datos atípicos, específicamente con el criterio de Dixon.

No se eliminaron datos mediante el criterio de exclusión citado. Se obtuvieron los resultados de los análisis estadísticos, en los que se obtuvo una generación per cápita promedio de $0.456 \mathrm{~kg} / \mathrm{hab} / \mathrm{d}$, una mediana de $0.395 \mathrm{~kg} / \mathrm{hab} / \mathrm{d}$ y una desviación estándar de $0.223 \mathrm{~kg} / \mathrm{hab} / \mathrm{d}$. El cálculo del tamaño real de la muestra dio un valor de 86 , por lo que se aceptaron las 91 premuestras con las cuales se trabajaron durante el estudio. Lo anterior, considerando un error muestral de $0.04 \mathrm{~kg} / \mathrm{hab} / \mathrm{d}$ y un percentil de la distribución $\mathrm{t}$ de student correspondiente al nivel de confianza de $90 \%$. Por último, el análisis de confiabilidad indicó que con el tamaño de muestra considerado, se tiene más del $90 \%$ de confianza en la aceptación de la hipótesis planteada.
CUADRO I. COMPONENTE DE LAS FRACCIONES DE LOS RESIDUOS SÓLIDOS URBANOS

\begin{tabular}{ll}
\hline Fracción & Componentes \\
\hline Orgánica & $\begin{array}{l}\text { Fracción de rápida biodegradabilidad } \\
\text { en donde se incluye a los residuos de } \\
\text { alimentos y jardinería, así como piezas } \\
\text { de madera. }\end{array}$ \\
\hline Papel y cartón & $\begin{array}{l}\text { Se incluye al papel de impresión, papel } \\
\text { revista o encerado, papel periódico, } \\
\text { además de cartón y cartón encerado. }\end{array}$ \\
\hline Plásticos & $\begin{array}{l}\text { En esta fracción se incorporaron } \\
\text { a los plásticos denominados PET, } \\
\text { HDPE,LDPE, PP, PS, PVC y mezclas de } \\
\text { ellos. }\end{array}$ \\
\hline Vidrio & $\begin{array}{l}\text { Se consideraron dos categorías: } \\
\text { transparente y color. }\end{array}$ \\
\hline Metales & $\begin{array}{l}\text { Se incluyen al aluminio en latas y perfil, } \\
\text { además de metales tanto en forma de } \\
\text { latas como en piezas. }\end{array}$ \\
\hline Peligrosos & $\begin{array}{l}\text { Se incluye a todos los materiales que } \\
\text { tengan características CRETIB, como } \\
\text { jeringas, baterías y medicamentos. }\end{array}$ \\
\hline Tecnológicos & $\begin{array}{l}\text { Se incluye todo aquel equipo o } \\
\text { pieza proveniente de algún aparato } \\
\text { electrodoméstico. }\end{array}$ \\
\hline $\begin{array}{l}\text { En esta fracción se incluye al papel } \\
\text { y toallas sanitarias, además de otros } \\
\text { subproductos como, hule, piezas de loza } \\
\text { y cerámica, materiales de la construcción } \\
\text { y finos. }\end{array}$ \\
\hline
\end{tabular}

${ }^{\text {a }} \mathrm{PET}=$ polietileno de tereftalato, HDPE $=$ polietileno de alta densidad, $\mathrm{LDPE}=$ polietileno de baja densidad, $\mathrm{PP}=$ polipropileno, $\mathrm{PS}=$ poliestireno, $\mathrm{PVC}=$ policloruro de vinilo

${ }^{\mathrm{b}}$ CRETIB $=$ corrosivo, reactivo, explosivo, toxico, inflamable y biológico infeccioso

\section{Generación de residuos no domésticos}

De acuerdo con la información descrita en el DENUE, la cabecera municipal de Berriozábal cuenta con 2092 establecimientos, los cuales pueden clasificarse en 4 unidades económicas generales (comercial, servicios, especiales y otros), como se observa en el cuadro II.

Es importante comentar que algunos de los establecimientos fueron eliminados del análisis debido a su repetición en diferentes unidades económicas. Por otro lado, también se descartaron aquellos locales o establecimientos que en la actualidad no tienen operación.

Para la obtención de la totalidad de los residuos generados en la fuente no doméstica, se realizó una sumatoria de la generación de RSU asignada a cada 


\section{CUADRO II. ESTABLECIMIENTOS Y UNIDADES ECONÓMICAS}

\begin{tabular}{ll}
\hline $\begin{array}{l}\text { Unidad } \\
\text { económica }\end{array}$ & Descripción \\
\hline Comercial & $\begin{array}{l}\text { En esta clasificación se encuentran establecimientos tanto pequeños como grandes, donde se comercializan bienes } \\
\text { de primera necesidad y otros. Entre los principales establecimientos se encuentran las tiendas de autoservicio, loca- } \\
\text { les comerciales de diversos tipos, así como tiendas de abarrotes y expendios de carnes y verduras. }\end{array}$ \\
\hline Servicios & $\begin{array}{l}\text { En esta clasificación se encuentran los establecimientos que brindan sostén a la sociedad, tanto desde el punto de } \\
\text { vista de transporte, como de realización de trámites y demás. Los establecimientos corresponden a oficinas públicas, } \\
\text { privadas, centros culturales y religiosos, además de gasolineras y hoteles. }\end{array}$ \\
\hline Especiales & Está integrada por centros educativos, unidades médicas y terminales terrestres. \\
\hline Otros & En esta última clasificación se agruparon a las áreas verdes de parques y jardines. \\
\hline
\end{tabular}

CUADRO III. GENERACIÓN DE RESIDUOS EN FUENTES NO DOMÉSTICAS

\begin{tabular}{|c|c|c|c|}
\hline Unidad económica & No. & $\begin{array}{l}\text { Generación } \\
(\mathrm{kg} / \mathrm{d})^{\mathrm{a}}\end{array}$ & $\begin{array}{l}\text { Generación } \\
\text { total }(\mathrm{t} / \mathrm{d})\end{array}$ \\
\hline Tienda de autoservicio & 1 & 637 & 0.64 \\
\hline Local comercial & 49 & 6.65 & 0.33 \\
\hline Tienda de abarrotes pequeña & 344 & 1.025 & 0.35 \\
\hline Expendio de carnes & 83 & 4.43 & 0.37 \\
\hline Expendio de frutas y verduras & 34 & 7.92 & 0.27 \\
\hline Varios & 1242 & 0.803 & 1.00 \\
\hline Infraestructura pública ${ }^{\mathrm{b}}$ & 49 & $\begin{array}{c}3.46 \mathrm{~kg} / \mathrm{local} / \mathrm{d} \\
0.43 \mathrm{~kg} / \mathrm{visitante} / \mathrm{d}\end{array}$ & 0.19 \\
\hline Oficinas pública/privada/cultural & 99 & 2.05 & 0.20 \\
\hline Restaurantes/alimentos & 123 & 14.96 & 1.84 \\
\hline Gasolineras & 1 & 53.12 & 0.05 \\
\hline Hoteles/posadas & 3 & 8.405 & 0.03 \\
\hline Centros educativos $^{\mathrm{c}}$ & 33 & 0.059/alumno/d & 0.23 \\
\hline Unidades médicas & 28 & $1.27 /$ consultorio/d & 0.04 \\
\hline Terminales terrestres & 3 & 2.103 & 0.01 \\
\hline Áreas verdes & $7778 \mathrm{~m}^{2}$ & $0.00993 \mathrm{~kg} / \mathrm{m}^{2} / \mathrm{d}$ & 0.077 \\
\hline Total & & & 5.61 \\
\hline
\end{tabular}

${ }^{\text {a }}$ Las unidades de la generación de residuos se encuentran en $\mathrm{kg} /$ establecimiento/d, a menos que se indique lo contrario

${ }^{\mathrm{b}}$ Los cálculos se realizaron considerando dos tasas de generación, dependiendo del tipo de infraestructura del que se trate

${ }^{\mathrm{c}}$ El cálculo se realizó considerando una estimación del número de alumnos en cada nivel educativo

tipo de establecimiento, con base en las referencias ya citadas de Heredia et al. (2007) y Alvarado et al. (2009) (Cuadro III).

\section{Generación per cápita doméstica, urbana y pro- yecciones}

Con los datos de la generación doméstica y no doméstica obtenidos anteriormente, se puede establecer que la cabecera municipal de Berriozábal, Chiapas, México, actualmente genera 21.34 t/d. Es decir, un equivalente a $0.619 \mathrm{~kg} / \mathrm{hab} / \mathrm{d}$ (Cuadro IV), cifra que se encuentra en el estándar de lo reportado por INECC (2012), ya que para ciudades de entre
CUADRO IV. GENERACIÓN DE RESIDUOS POR FUENTE GENERADORA

\begin{tabular}{lcc}
\hline Fuente de generación & $\begin{array}{c}\text { Generación per } \\
\text { cápita }(\mathrm{kg} / \mathrm{hab} / \mathrm{d})\end{array}$ & $\begin{array}{c}\text { Cantidad } \\
(\mathrm{t} / \mathrm{d})\end{array}$ \\
\hline $\begin{array}{l}\text { Casa habitación } \\
\text { Residuos de comercios }\end{array}$ & 0.456 & 15.73 \\
y servicios públicos & 0.160 & 5.61 \\
Total & $\mathbf{0 . 6 1 9}$ & $\mathbf{2 1 . 3 4}$ \\
\hline
\end{tabular}

Nota: las cifras de residuos sólidos urbanos están calculadas al año 2016 y fueron obtenidas mediante proyecciones realizadas con el método geométrico y una población para este mismo año de 34491 hab 


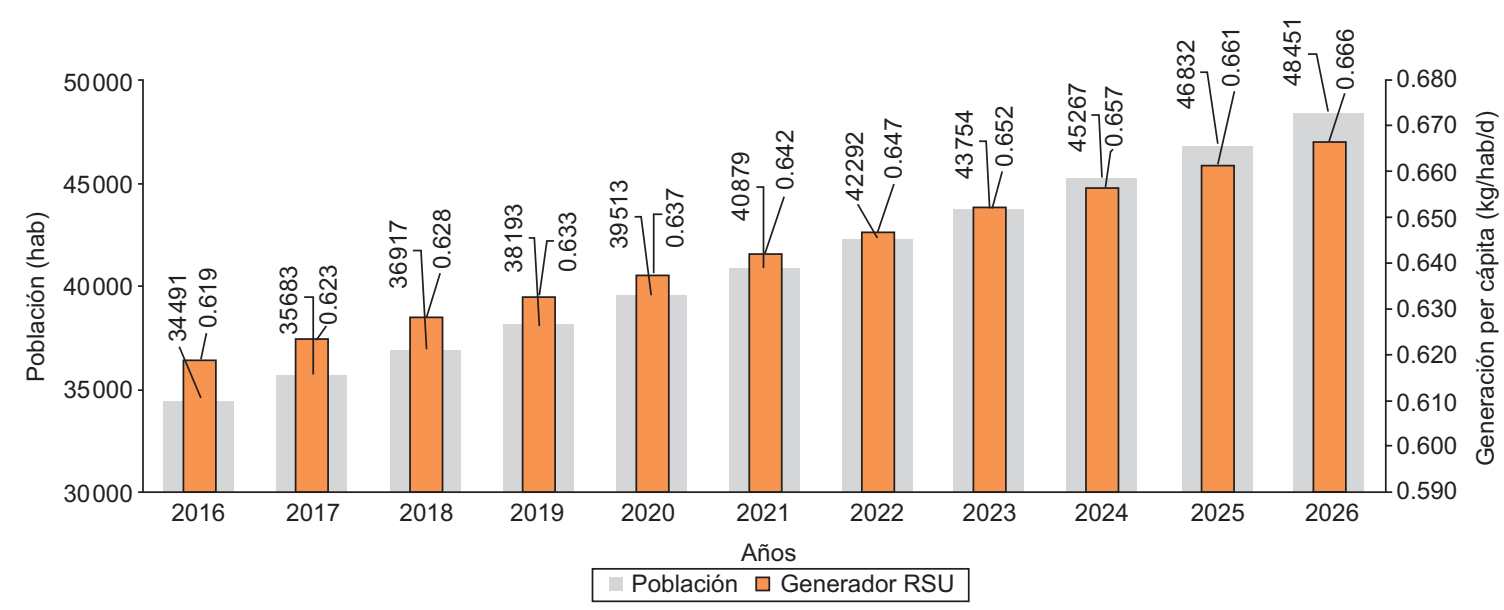

Fig. 2. Población y generación per cápita de residuos sólidos urbanos (RSU) en Berriozábal 2016 - 2026 Fuente: Las proyecciones se realizaron con el método geométrico, considerando una tasa de crecimiento poblacional anual del $3.45 \%$ y de $1 \%$ para la generación per cápita

\section{Clasificación de subproductos}

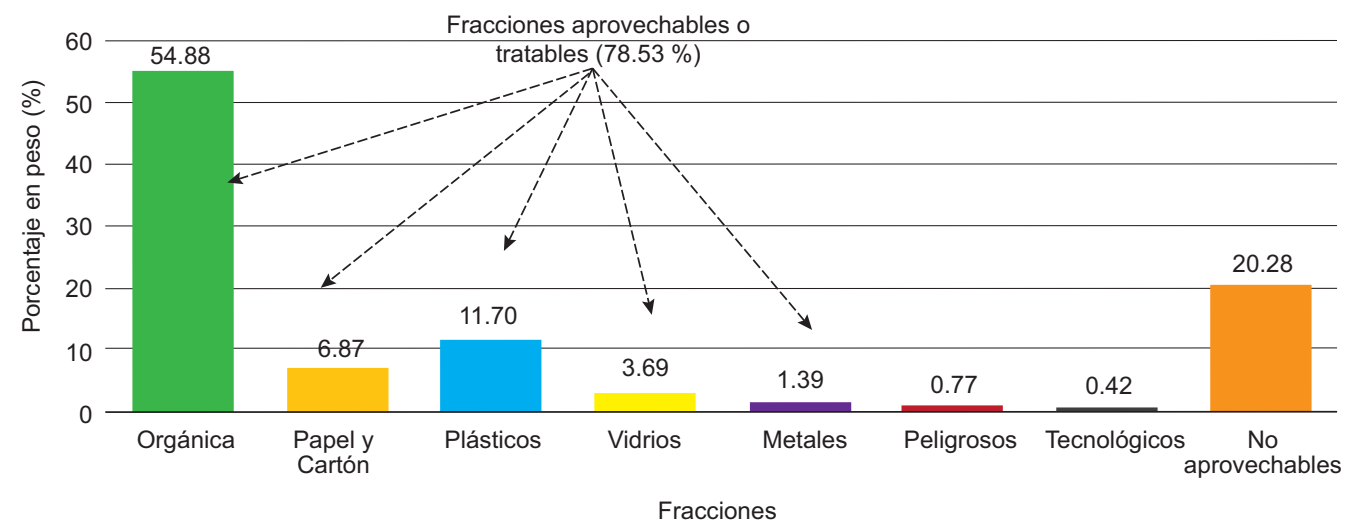

Fig. 3. Fracciones de los residuos sólidos urbanos generadas en Berriozábal, Chiapas, México

20 y 30 mil habitantes, indica que $0.60 \mathrm{~kg} / \mathrm{hab} / \mathrm{d}$ es la tasa generada en promedio.

Por otro lado, una proyección a futuro de la tasa de generación de RSU y del crecimiento poblacional (Fig. 2) permite ver que en un periodo de 10 años, la cabecera municipal de Berriozábal, Chiapas, estará generando $0.66 \mathrm{~kg} / \mathrm{hab} / \mathrm{d}$. Lo que se traduce en $32.29 \mathrm{t} / \mathrm{d}$, por lo que será necesario replantear las estrategias de manejo actual de RSU. Inclusive, considerar una reforma a la normatividad o legislación local a favor de la separación de residuos en casa habitación. Lo anterior, debido a que actualmente el municipio no se da abasto con la recolección de residuos por la falta de equipamiento. Por lo que únicamente se está recolectando $63.5 \%$ de los RSU generados (SEMAHN 2013).

\section{Composición de los RSU en Berriozábal}

Respecto a la composición de residuos, la figura 3 y el cuadro $\mathbf{V}$ muestran que la fracción orgánica se produce en gran medida, con los porcentajes más altos en los residuos de alimentos (42.44\%), seguido de los residuos de jardín (12.14\%).

Otros subproductos como los plásticos (11.70\%) siguen en aumento, particularmente los llamados polietileno de tereftalato (PET) con $1.27 \%$, polietileno de alta densidad (HDPE) con $2.78 \%$ y polietileno de baja densidad (LDPE) con $4.41 \%$. Dicho aumento está motivado por las tendencias de consumo actuales, específicamente la de "usar y tirar".

Se destaca también que de la totalidad de los residuos, poco más de $78 \%$ son materiales susceptibles de recuperación, por lo que podrían ser aprovechados 
CUADRO V. COMPOSICIÓN DE LOS RESIDUOS SÓLIDOS DOMÉSTICOS DE BERRIOZÁBAL, CHIAPAS, MÉXICO

\begin{tabular}{|c|c|c|}
\hline Fracción & Componentes & $\begin{array}{l}\% \text { en } \\
\text { peso }\end{array}$ \\
\hline \multirow[t]{3}{*}{ Orgánica } & Residuos alimenticios & 42.44 \\
\hline & Residuos de jardín & 12.14 \\
\hline & Madera & 0.30 \\
\hline \multirow{5}{*}{$\begin{array}{l}\text { Papel y } \\
\text { cartón }\end{array}$} & Cartón & 3.20 \\
\hline & Cartón encerado/Tetrapak & 1.11 \\
\hline & Papel encerado/revista & 0.64 \\
\hline & Papel de impresión & 0.79 \\
\hline & Papel periódico & 1.14 \\
\hline \multirow[t]{6}{*}{ Plásticos } & HDPE & 2.78 \\
\hline & LDPE & 4.41 \\
\hline & PET & 1.27 \\
\hline & Plásticos varios & 2.45 \\
\hline & PVC & 0.12 \\
\hline & PS & 0.67 \\
\hline \multirow[t]{2}{*}{ Vidrios } & Vidrio de color & 0.20 \\
\hline & Vidrio transparente & 3.49 \\
\hline \multirow[t]{3}{*}{ Metales } & Aluminio & 0.07 \\
\hline & Latas de otros metales & 0.96 \\
\hline & Material ferroso & 0.37 \\
\hline Peligrosos & Jeringas, pilas, medicamentos, etc. & 0.77 \\
\hline Tecnológicos & Equipos o piezas electrónicas & 0.42 \\
\hline No & Pañal desechables y toallas sanitarias & 8.40 \\
\hline \multirow[t]{8}{*}{ aprovechable } & Papel sanitario & 6.77 \\
\hline & Cuero/zapatos de & 0.11 \\
\hline & Trapo (natural y sintético) & 2.72 \\
\hline & Loza y cerámica & 0.72 \\
\hline & Hule & 0.27 \\
\hline & Residuos de la construcción & 0.93 \\
\hline & Residuos finos & 0.33 \\
\hline & Algodón & 0.03 \\
\hline
\end{tabular}

PET $=$ polietileno de tereftalato, $\mathrm{HDPE}=$ polietileno de alta densidad, $\mathrm{LDPE}=$ polietileno de baja densidad, $\mathrm{PS}=$ poliestireno, $\mathrm{PVC}=$ policloruro de vinilo

de alguna manera para no enviarlos directamente a disposición final y con ello alargar la vida útil de los sitios de depósito de residuos.

En menor medida aparecen los residuos peligrosos y tecnológicos (también llamados eléctricos y electrónicos) con $1.19 \%$ ambos. Los cuales en un futuro requerirán de algún mecanismo de control o tratamiento, dada las altas cantidades que podrían generarse en los años próximos.

Por otro lado, el peso volumétrico fue de $162.48 \mathrm{~kg} / \mathrm{m}^{3}$, valor alto respecto a los $153.12 \mathrm{~kg} / \mathrm{m}^{3}$ reportados por el INECC (2012) como promedio nacional. Lo anterior, debido posiblemente a la época del año en que se realizó el estudio, que fue al aparecer las primeras lluvias fuertes, lo que incorporó agua o humedad a las muestras de residuos.

\section{CONCLUSIONES}

Se aplicó un método estadístico para obtener la tasa de generación per cápita en fuentes domésticas, la cual fue de $0.456 \mathrm{~kg} / \mathrm{hab} / \mathrm{d}$. Este método es de los más empleados a nivel nacional por su confiabilidad, aunque no es el único. Existen también balances de masa, análisis de peso-volumen, análisis de cargas y técnicas asociativas, esta última muy similar a la denominada Evaluación Rápida de Fuentes de Contaminación de Aire, Agua y Suelo (ERFCA).

La generación de residuos en fuentes no domésticas arrojó un valor de $0.16 \mathrm{~kg} / \mathrm{hab} / \mathrm{d}$, que en conjunto con la generación per cápita doméstica equivalen a $0.619 \mathrm{~kg} / \mathrm{hab} / \mathrm{d}$. Estas cifras aumentarán en los próximos años, influenciados por varios factores, como el aumento de conjuntos habitacionales en la cabecera municipal de Berriozábal. En este sentido, el H. Ayuntamiento tendrá que actuar para aumentar las coberturas de recolección de RSU, ya sea al adquirir un mayor número de vehículos o al implementar otras estrategias de carácter normativo.

Respecto a la composición de los RSU y sus porcentajes, se destaca que la generación sigue siendo gran medida de tipo orgánica, con valores por encima de $50 \%$. Aunque la diversidad y altos porcentajes de otros subproductos como los plásticos, confirma la tendencia mencionada en el trabajo de Calva y Rosas (2014), que es la de un cambio en los patrones de consumo de la población de México.

Otros subproductos como los residuos peligrosos y tecnológicos, no representan en la actualidad un riesgo por los bajos porcentajes de aparición (1.19 $\%$ ambos), aunque en un futuro será necesario que las autoridades municipales regulen su manejo y disposición. Lo anterior, debido a las mezclas e interacciones de componentes en los residuos, lo que ocasiona que los RSU adquieran cierto grado de peligrosidad.

En términos de gestión y manejo de residuos, es importante que las autoridades municipales se acerquen a la academia, a fin de desarrollar una mayor cantidad de estudios, actualizar cifras de generación de residuos, realizar diagnósticos ambientales o incluso, obtener orientación en el diseño de las obras de ingeniería o en la adquisición de equipos de colecta. 


\section{AGRADECIMIENTOS}

Esta investigación fue realizada gracias al Programa Universidad Nacional Autónoma de México - Dirección General de Asuntos del Personal Académico - Programa de Apoyo a Proyectos de Investigación e Innovación Tecnológica (UNAM-DGAPA-PAPIIT IN105516). Asimismo, se agradece a los revisores por sus útiles críticas y sugerencias.

\section{REFERENCIAS}

Aguilar Q, Armijo C., Taboada P. y Aguilar X. (2010). Potencial de recuperación de residuos sólidos domésticos dispuestos en un relleno sanitario. Rev. Ing. (32), 16-27. DOI: $10.16924 \% 2$ Friua.v0i32.190

Al-Jarallah R. y Aleisa E. (2014). A baseline study characterizing the municipal solid waste in the State of Kuwait. Waste Manage. 34 (5), 952-960.

DOI: 10.1016/j.wasman.2014.02.015

Alvarado H., Nájera H., González F. y Palacios R. (2009). Estudio de generación y caracterización de los residuos sólidos domiciliarios en la cabecera municipal de Chiapa de Corzo, Chiapas, México. Revista Lacandonia 3 (1), 85-92.

Araiza J., López C. y Ramírez N. (2015). Manejo de residuos sólidos urbanos: caso de estudio en Las Margaritas, Chiapas. Revista AIDIS de Ingeniería y Ciencias Ambientales: Investigación, Desarrollo y Práctica 8 (3), 299-311.

Bernache G., Sánchez S., Garmendia A., Dávila A. y Sánchez M. (2001). Solid waste characterization study in the Guadalajara metropolitan zone, Mexico. Waste Manag. Res. 19, 413-424.

DOI: $10.1177 / 0734242 X 0101900506$

Buenrostro O. y Bocco G. (2003). Solid waste management in municipalities in Mexico: goals and perspectives. Resour. Conserv. Recy. 39 (3), 251-263. DOI: $10.1016 / \mathrm{S} 0921-3449(03) 00031-4$

Calva C. y Rosas R. (2014). Diagnóstico de la gestión de residuos sólidos urbanos en el municipio de Mexicali, México: retos para el logro de una planeación sustentable. Información Tecnológica 25 (3), 59-72.

DOI: $10.4067 / \mathrm{S} 0718-07642014000300009$

Castillo E. y De Medina L. (2014). Generación y composición de residuos sólidos domésticos en localidades urbanas pequeñas en el estado de Veracruz, México. Rev. Int. Contam. Ambie. 30 (1), 81-90.

Carrera J. (2012). Estudio de generación y caracterización de residuos sólidos domiciliarios en 3 localidades del Municipio de Juárez, Chiapas. Tesis de Licenciatura. Escuela de Ingeniería Ambiental, Universidad de Ciencias y Artes de Chiapas, Reforma, Chiapas, México, $107 \mathrm{pp}$.

Cabrera A. (2013). Caracterización de residuos sólidos no peligrosos en la central hidroeléctrica Belisario Domínguez. Informe Técnico. Escuela de Ingeniería Ambiental, Universidad de Ciencias y Artes de Chiapas, Tuxtla Gutiérrez, Chiapas, México, 70 pp.

DOF (2014). Ley general para la prevención y gestión integral de los residuos. Diario Oficial de la Federación. 8 de octubre de 2003.

Escamirosa L., Del Carpio C., Castañeda G. y Quintal C. (2001). Manejo de los residuos sólidos domiciliarios en la ciudad de Tuxtla Gutiérrez, Chiapas. Plaza y Valdés Editores, Ciudad de México, México, 129 pp.

Esquinca F., Escobar J., Hernández A. y Villalobos J. (1996). Caracterización y generación de los residuos sólidos de Tuxtla Gutiérrez, Chiapas, Memorias. XXV Congreso Interamericano de Ingeniería Sanitaria y Ambiental. Ciudad de México. Del 3 al 7 de noviembre. Versión digital.

Esquinca F., Escobar J., Hernández A., Sánchez G. y Suárez H. (1997). Estudios de caracterización y generación de residuos sólidos municipales de 5 localidades de la costa del estado de Chiapas, Memorias. XI Congreso Nacional de Ingeniería Sanitaria y Ciencias Ambientales. Zacatecas. Del 4 al 7 de noviembre. Versión digital.

Fierro A., Armijo C., Buenrostro O. y Valdez B. (2010). Análisis de la generación de residuos sólidos en supermercados de la ciudad de Mexicali, México. Rev. Int. Contam. Ambie. 26 (4), 291-297.

GTZ (2003). La Basura en el limbo: desempeño de gobiernos locales y participación privada en el manejo de residuos urbanos. Agencia de Cooperación Técnica Alemana (Gesellschaft für Technische Zusammenarbeit) - Comisión Mexicana de Infraestructura Ambiental. Manual. México, D.F., 98 pp.

Heredia P., Sánchez J., Rodríguez M. y Aguilar R. (2007). Guía para la revisión de proyectos ejecutivos, planes de regularización o evaluación de la conformidad según la NOM-083-SEMARNAT-2003. Secretaría de Medio Ambiente y Recursos Naturales (SEMARNAT) - Agencia de Cooperación Técnica Alemana (GTZ, por sus siglas en alemán). Manual. México, D.F., 109 pp.

INE (1997). Estadísticas e indicadores de inversión sobre residuos sólidos municipales en los principales centros urbanos de México. Instituto Nacional de Ecología. México, D.F., 59 pp.

INECC (2012). Diagnóstico básico para la gestión integral de los residuos. Instituto Nacional de Ecología y Cambio Climático (INECC)-Secretaría de Medio Ambiente y Recursos Naturales (SEMARNAT). México, D.F., 201 pp. 
INEGI (2016). Directorio Estadístico Nacional de Unidades Económicas (DENUE) [en línea]. http://www3. inegi.org.mx/sistemas/descarga/?c=200 10/06/16.

INE-SEMARNAT (1999). Minimización y manejo ambiental de los residuos sólidos. Instituto Nacional de Ecología - Secretaría de Medio Ambiente y Recursos Naturales México, D.F., 236 pp.

INE-SEMARNAT (2006). Diagnóstico básico para la gestión integral de los residuos. Instituto Nacional de Ecología - Secretaría de Medio Ambiente y Recursos Naturales. México, D.F., 112 pp.

Ojeda S. y Beraud J.L. (2003). The municipal solid waste cycle in Mexico: final disposal. Resour. Conserv. Recy. 39 (3), 239-250.

DOI: $10.1016 / \mathrm{S} 0921-3449(03) 00030-2$

Reyes J. (2016). Estudio de generación y caracterización de los residuos sólidos domiciliarios (RSD) en la localidad de la Independencia municipio de La Concordia, Chiapas, México. Informe Técnico. Escuela de Ingeniería Ambiental, Universidad de Ciencias y Artes de Chiapas, Tuxtla Gutiérrez, Chiapas, 70 pp.

SECOFI (1985a). Norma mexicana NMX-AA-015-1985. Protección al ambiente - contaminación del suelo residuos sólidos municipales - muestreo - método de cuarteo. Dirección general de normas. Secretaría de comercio y fomento industrial. Diario oficial de la federación, México, 18 marzo de 1985.

SECOFI (1985b). Norma mexicana NMX-AA-019-1985. Protección al ambiente - contaminación del suelo - residuos sólidos municipales - peso volumétrico "in situ". Dirección general de normas. Secretaría de comercio y fomento industrial. Diario oficial de la federación, México, 18 marzo de 1985.

SECOFI (1985c). Norma mexicana NMX-AA-022-1985. Protección al ambiente - contaminación del suelo - residuos sólidos municipales - selección y cuantificación de subproductos. Dirección general de normas. Secretaría de comercio y fomento industrial. Diario oficial de la federación, México, 18 marzo de 1985.

SECOFI (1985d). Norma mexicana NMX-AA-061-1985. Protección al ambiente - contaminación del suelo - residuos sólidos municipales - determinación de generación. Dirección general de normas. Secretaría de comercio y fomento industrial. Diario oficial de la federación, México, 8 agosto de 1985.

SEMAHN (2013). Diagnóstico estatal de los residuos sólidos en los municipios de Chiapas. Secretaría de Medio Ambiente e Historia Natural [en línea]. http:// www.semahn.chiapas.gob.mx/portal/residuos/diagnostico 10/06/16.

SHCP (2011). Programa regional de desarrollo: región I metropolitana. Secretaría de Hacienda y Crédito Público - Subsecretaría de Planeación, Presupuestos y Egresos. Informe. Tuxtla Gutiérrez, Chiapas, México, $53 \mathrm{pp}$.

Villalobos J. (1996). Manejo y disposición final de los residuos sólidos municipales en la ciudad de Tuxtla Gutiérrez, Chiapas. Tesis de Maestría. Facultad de Ingeniería Civil, Universidad Autónoma de Nuevo León, México, 301 pp. 